\title{
Identification of Atrial Fibrillation Episodes Using a Camera as Contactless Sensor
}

\author{
Valentina D.A. Corino ${ }^{1}$, Luca Iozzia ${ }^{1}$, Andrea Mariani ${ }^{1}$, Giacomo D’Alessandro ${ }^{1}$, Claudia \\ D’Ettorre $^{1}$, Luca Cerina ${ }^{1}$, Giorgio Scarpini ${ }^{2}$, Federico Lombardi $^{2}$, Luca T. Mainardi $^{1}$ \\ ${ }^{1}$ Dipartimento di Elettronica, Informazione e Bioingegneria, Politecnico di Milano, Milan, Italy \\ ${ }^{2}$ Fondazione IRCCS Ca Granda Ospedale Maggiore Policlinico, U.O.C. di Malattie Cardiovascolari, \\ Universitá degli Studi di Milano, Dipartimento di Scienze Cliniche e di Comunitá, Milan, Italy
}

\begin{abstract}
Identification of paroxysmal atrial fibrillation $(A F)$ can be difficult and undiagnosed AF patients are at high risk of cardioembolic stroke or other complications associated with AF. The aim of this study is to analyze the video photoplethysmografic (vPPG) signal obtained from a videocamera to explore the possibility of discriminating AF from normal sinus rhythm (NSR) and other arrhythmias (ARR). We acquired 24 3-min long face-videos (8for each rhythm) using an industrial camera. After preprocessing, vPPG signal was extracted using zero-phase component analysis. Diastolic minima were detected and inter-diastolic series obtained. The signals were characterized by time domain indexes, the sample entropy (SampEn); and the shape similarity index (ShapeSim). The time domain indexes and ShapeSim are significantly different when comparing the group of patients with AF or ARR to subjects in NSR. SampEn is significantly higher in AF than in NSR and ARR. From the shape analysis, it can be noted that waves in NSR are more similar than in AF. These preliminary results show the capability of different indexes to capture differences among AF, ARR and NSR. Further studies will help in assessing the performance of the $v P P G$ signal to screen general population.
\end{abstract}

\section{Introduction}

In the recent years new monitoring methods have been presented in the literature to detect atrial fibrillation (AF) events by the use of ubiquitous devices such as smartphone [1-3] or wristband device [4]. AliveCor is a single-lead rhythm strip that, combined with a smartphone, is able to detect episodes of AF with $98 \%$ of sensitivity. The company Preventicus arises with an app that uses the back camera of the smarphone to individualize AF episodes by the analysis of finger photoplethysmographic (PPG) signal. Another investigation group uses the same technology to discriminate between $\mathrm{AF}$, sinus rhythm (SR), premature atrial contractions and premature ventricular contractions. The results seemed quite promising, demonstrating a sensitivity of 0.970 , specificity of 0.935 , and accuracy of 0.951 for real-time identification of an irregular pulse during AF. The app also showed good accuracy for premature atrial contractions 0.955 and premature ventricular contractions discrimination 0.960 [2]. In the video PPG (vPPG) technology, the study of Couderc [5] was the first one that demonstrated the feasibility of AF detection by the use of a contactless technology. The present study aims to enlarge Couderc's study by introducing the detection of other arrhythmias (ARR) that may have the same dangerous consequences of AF.

\section{Materials and Methods}

\subsection{Protocol}

Twenty-four patients were enrolled at Ospedale Maggiore Policlinico of Milan, Italy. The study was approved by the internal Ethical Committee of the Hospital. Each patient was asked to sit on a chair and to move as less as possible. The ambient setting included sunlight coming from a window, placed in front of subject's face to avoid possible shadows on his face and an artificial light of neon lamps of the room.

An industrial camera (spatial resolution of $659 \times 494$ pixels) positioned on a tripod, was placed in front of the subject at a distance of $1.5 \mathrm{~m}$ and connected to a PC workstation. The camera recorded the face of the patient for the whole session of the experiment that lasted 3 minutes. During the acquisition, a 12 leads ECG recorder was used to allow a clinician to determine the type of rhythm of the subject according to three different classes: SR, AF and ARR. According to the clinician classification, 8 patients were in SR, 8 in AF and 8 in ARR during the video recording. Videos were acquired with a frame-rate of 120 frames 
per second (FPS) and 8-bits resolutions. Data were saved in RGB, uncompressed, AVI raw format to be processed offline. The camera was equipped with $15 \mathrm{~mm}$ fixed focal length lenses.

\subsection{Extraction of vPPG}

The video recordings were saved in $\mathrm{RGB}$ raw format and processed offline to derive the vPPG signal. The video preprocessing includes the three following steps: i) face detection; ii) motion flow; iii) signal extraction.

Face detection and motion flow: Face detection was performed in the first frame by Viola-Jones algorithm[6] and refreshed every $30 \mathrm{~s}$ to bootstrap the face tracker [7]. Successively, the face tracking was implemented by using Lucas-Kanade-Tomasi algorithm [8], consisting of two steps: one to choose the best features for tracking and one to align the images. In each frame three regions of interest were considered: forehead $\left(\mathrm{ROI}_{1}\right)$, nose $\left(\mathrm{ROI}_{2}\right)$ and cheek $\left(\mathrm{ROI}_{3}\right)$. These preprocessing steps were implemented in OpenCV.

Signal extraction: A spatial average of pixels intensity was calculated for each ROI in order to have $N_{\text {channels }} \times$ $N_{R O I}$ time series. Briefly, within each ROI a spatial average of pixel intensities of each RGB channel was calculated for each frame to generate $N$ raw signals (where $N$ is the number of color channels). The temporal series were first detrended using the technique described on [9] setting the smoothness parameter $\lambda=400$. A hamming bandpass filter with cutoff frequencies of $f_{1}=0.1, f_{2}=5 \mathrm{~Hz}$ was applied to remove high frequencies correlated mainly to artificial light and low frequencies associated to slow movements.

After this preprocessing, to enhance the pulsatile component of the reflected light and reduce the motion noise, the vPPG signal was extracted using the zero-phase component analysis (ZCA) [7]. Since each ROI returns a vPPG signal, the selection of the signal to use for further analysis has been made according to the highest SNR, computed as

$$
S N R=10 * \log _{10}\left(\frac{\int_{f_{1}}^{f_{2}} \mathrm{PSD}(\mathrm{f}) \mathrm{df}}{\int_{0.1}^{f_{1}} \mathrm{PSD}(\mathrm{f}) \mathrm{df}+\int_{\mathrm{f}_{2}}^{4} \mathrm{PSD}(\mathrm{f}) \mathrm{df}}\right)
$$

where $f_{1}=f_{p}-0.15, f_{2}=f_{p}+0.15$ and $f_{p}$ is the pulse frequency (measured in $\mathrm{Hz}$ ) identified as the highest peak in the power spectral density (PSD).

Figure 1 shows an example of vPPG from three patients in the three rhythms.

As fiducial point for the extraction of pulse-to-pulse interval series, the vPPG diastolic peaks detected by the algorithm of Scholkmann et al. [10] were considered.

\subsection{Signal characterization}

To characterize the type of rhythm of each patient, different features have been extracted from vPPG signal: three belonging to time domain, sample entropy (Sam$\mathrm{pEn}$ ), and the shape similarity index (ShapeSim).

Time domain parameters and SampEn were computed on the inter-diastolic interval series, whereas shape analysis to obtain the ShapeSim index was performed on the vPPG signal.

Variability analysis of the inter-diastolic interval series includes the mean (M), the standard deviation (SD), the root of the mean squared differences of successive intervals, (rMSSD) and the percentage of interval differences of successive intervals greater than $50 \mathrm{~ms}$ (pNN50) [11].

Irregularity of the inter-diastolic interval series was assessed by sample entropy (SampEn), that quantifies the unpredictability of fluctuations. The presence of repetitive patterns of fluctuation in a time series makes it more predictable than a time series in which such patterns are absent. SampEn reflects the likelihood that similar patterns of observations will not be followed by additional similar observations. SampEn is the negative natural logarithm of the conditional probability that two sequences of length $\mathrm{m}$ that match within tolerance $r$ will also match at the $m+$ 1 length. Defining as A the total number of matches of length $m+1$ and B the total number of matches of length $m$, SampEn is computed as [12]

$$
\text { SampEn }=-\ln (A / B)=-\ln (A)+\ln (B)
$$

In this study, $m$ was used equal to 1 and $r$ equal to 0.25 times the standard deviation of the series, as commonly used.

To assess wave similarity, each wave is represented as a point of the p-dimensional real space, the normalized waves are points belonging to the p-dimensional unitary sphere. Hence, the morphological dissimilarity between two waves is evaluated by using the standard metric of the sphere to compute their distance [13]

$$
D_{i, j}=\arccos \left(\mathbf{w}_{\mathbf{i}}^{\mathbf{N}} \cdot \mathbf{w}_{\mathbf{j}} \mathbf{N}^{\mathbf{N}}\right)
$$

where $\mathbf{w}_{\mathbf{i}}$ and $\mathbf{w}_{\mathbf{j}}$ represent the $i$ th and $j$ th normalized waves, and $(\cdot)$ denotes the scalar product. A measure of similarity between waves is obtained by calculating the relative number of similar pairs of waves in the signal. The similarity depends on the threshold used in evaluating the similarity, that is, two waves are considered to be similar when their distance is lower than the threshold. In this study, one pulse in the vPPG signal is considered as wave and the threshold was set to $\pi / 2$. 
(a)

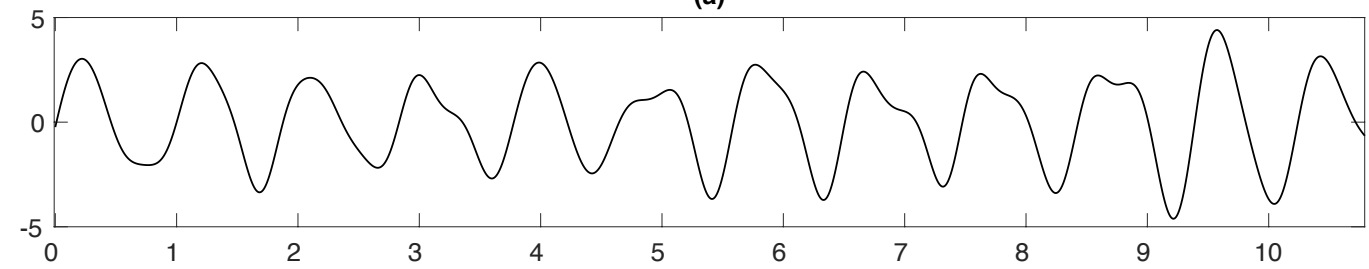

(b)

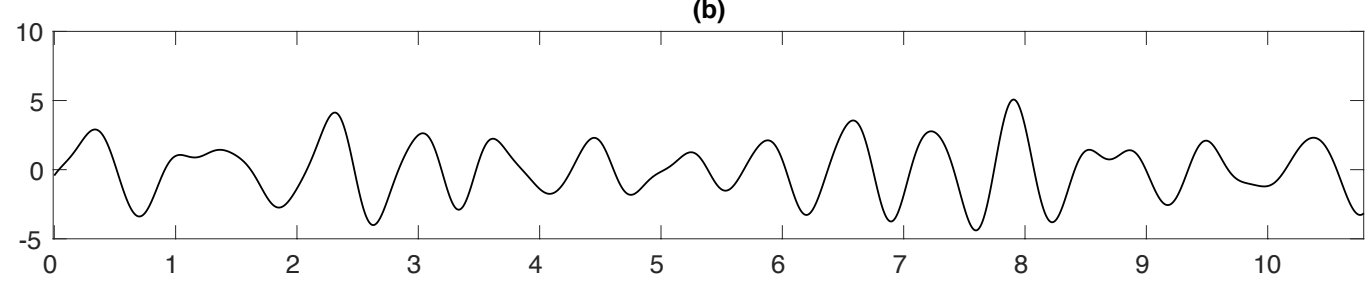

(c)

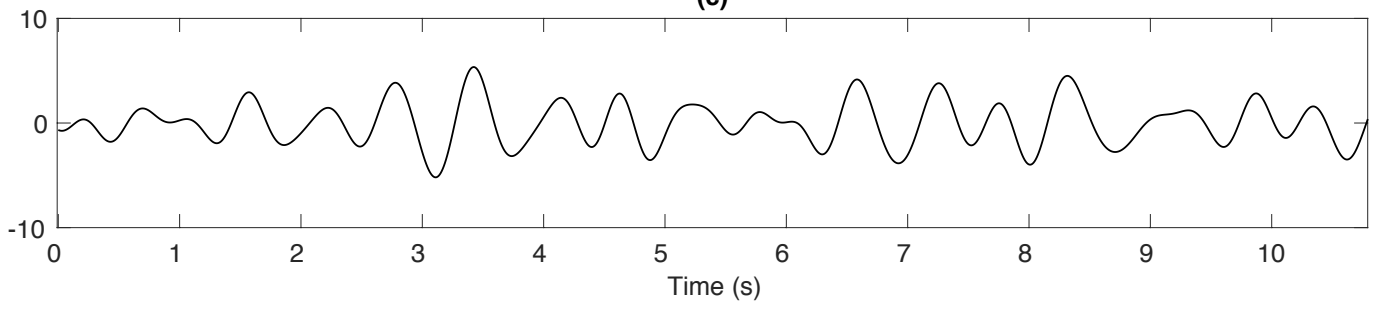

Figure 1. Example of vPPG extracted during (a) sinus rhythm, (b) atrial fibrillation and (c) other arrhythmias.

\subsection{Statistical analysis}

Kruskal-Wallis one-way analysis of variance was performed to compare the computed parameters during NSR, AF and ARR. If the p-value of the Kruskal-Wallis test was significant, an unpaired Wilcoxon test with Holms correction was applied. A p $<0.05$ was considered statistically significant. All analyses and statistical tests were performed using MATLAB R2016a (The MathWorks, USA).

\section{Results}

Figure 2 shows the boxplots of the computed parameters during the three rhythms. All variability parameters can differentiate between NSR and AF, being the variability of inter-diastolic interval series significantly higher in AF than in SR. Two variability parameters (SD and rMSSD) are significantly different also comparing NSR and ARR, being the variability of inter-diastolic interval series significantly higher in ARR than in NSR. SampEn is the only parameter that is significantly different comparing AF with ARR and NSR, being SampEn higher in AF than in NSR and ARR. From the shape analysis, ShapeSim is higher in NSR than in AF and ARR, i.e., waves in NSR are more similar than in AF and ARR.

\section{Conclusions}

These preliminary results show the capability of different indexes to capture differences among AF, ARR and NSR. Further studies will help in assessing the performance of the vPPG signal to screen general population.

\section{References}

[1] Krivoshei L, Weber S, Burkard T, Maseli A, Brasier N, Khne M, Conen D, Huebner T, Seeck A, Eckstein J.Smart detection of atrial fibrillation. Europace 2017;19:753-757.

[2] McManus DD, Chong JW, Soni A, Saczynski JS, Esa N, Napolitano C, Darling CE, Boyer E, Rosen RK, Floyd KC, Chon KH. PULSE-SMART: Pulse-Based Arrhythmia Discrimination Using a Novel Smartphone Application. J Cardiovasc Electrophysiol. 2016;27:51-7.

[3] Lau JK, Lowres N, Neubeck L, Brieger DB, Sy RW, Galloway CD, Albert DE, Freedman SB. iPhone ECG application for community screening to detect silent atrial fibrillation: A novel technology to prevent stroke. Int J Cardiol 2013;165:193-4.

[4] Corino VDC, Laureanti R, Ferranti L, Scarpini G, Lombardi F, Mainardi LT. Detection of atrial fibrillation episodes using a wristband device. Physiol Meas 2017;38:787-799.

[5] Couderc JP, Kyal S, Mestha LK, Xu B, Peterson DR, Xia 

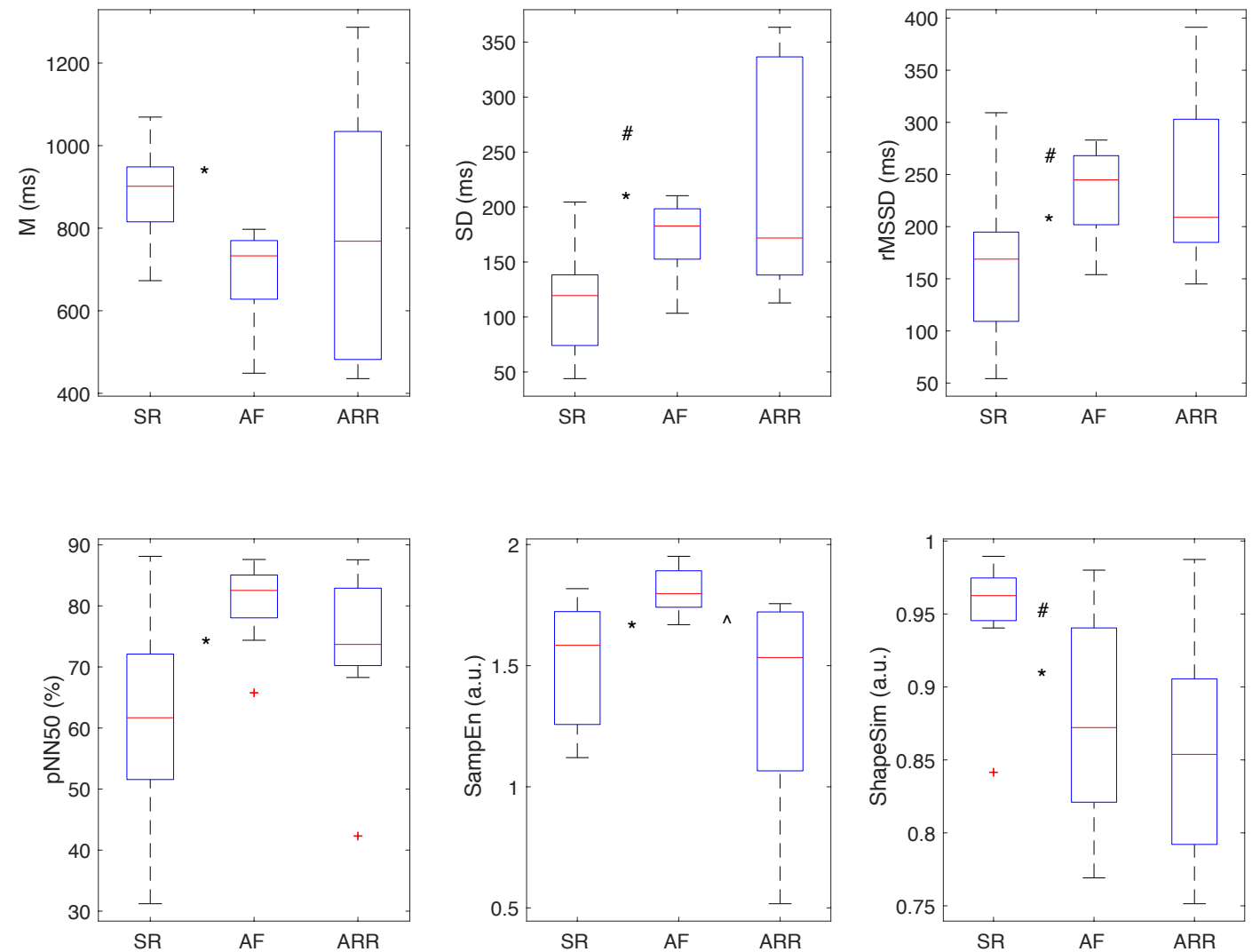

Figure 2. Boxplots of the computed parameters during sinus rhythm (SR), atrial fibrillation (AF) and other arrhythmias (ARR). $* \mathrm{p}<0.05$ AF vs. NSR; $\wedge \mathrm{p}<0.05$ AF vs. ARR; \# $\mathrm{p}<0.05$ NSR vs. ARR.

X, Hall B. Detection of atrial fibrillation using contactless facial video monitoring. Heart Rhythm 2015;12:195-201.

[6] Viola P, Jones M. Rapid object detection using a boosted cascade of simple features. Proceedings of IEEE Conference on Computer Vision and Pattern Recognition. 2001; 511: 1-9.

[7] Iozzia L, Cerina L, Mainardi LT. Relationships between heart-rate variability and pulse-rate variability obtained from video-PPG signal using ZCA. Physiol Meas 2016;37:1034-44.

[8] Lucas BD, Kanade T. An iterative image registration technique with an application to stereo vision. Proceedings of the 7th International Joint Conference on Artificial Intelligence 1981; 674-679.

[9] Tarvainen MP, Ranta-Aho PO, Karjalainen PA. An advanced detrending method with application to HRV analysis. IEEE Trans Biomed Eng 2002;44:172-5.

[10] Scholkmann F, Boss J, Wolf M. An efficient algorithm for automatic peak detection in noisy periodic and quasiperiodic signals. Algorithms 2012;5:588-603.

[11] Task Force of the European Society of Cardiology and the North American Society of Pacing and Electrophysiology. Heart Rate Variability. Eur Heart J 1996;17:354-381.

[12] Richman JS, Moorman JR. Physiological time-series analysis using approximate entropy and sample entropy. Am J Physiol Heart Circ Physiol 2000;278:H2039-49.

[13] Faes L, Nollo G, Antolini R, Gaita F and Ravelli F. A method for quantifying atrial fibrillation organization based on wave-morphology similarity IEEE Trans Biomed Eng 2002;49:1504-13.

Address for correspondence:

Valentina D.A. Corino via Golgi 39, 20133 Milan (Italy)

valentina.corino@polimi.it 